15th AlAA Computational Fluid

Dynamics Conference

11-14 June 2001 Anaheim, CA

\title{
DEVELOPMENT AND VALIDATION OF SOLUTION-ADAPTIVE, PARALLEL SCHEMES FOR COMPRESSIBLE PLASMAS
}

\author{
K. G. Powell, G. Tóth†, D. L. De Zeeuw; P. L. Roe \\ T. I. Gombosi Tand Q. F. Stout" \\ University of Michigan, Ann Arbor, Michigan 48109, U.S.A.
}

\section{Abstract}

Techniques that have become common in aerodynamics codes have recently begun to be implemented in space-physic codes, which solve the governing equations for a compressible plasma. These techniques include high-resolution upwind schemes, block-based solution-adaptive grids and domain decomposition for parallelization. While some of these techniques carry over relatively straightforwardly from aerodynamics to space physics, space physics simulations pose some new challenges. This paper gives a brief review of the state-of-the-art in modern space-physics codes, including a validation study of several of the techniques in common use. A remaining challenge is that of flows that include regions in which relativistic effects are important; some background and preliminary re-

\footnotetext{
${ }^{*}$ Professor, Aerospace Engineering, AIAA Senior Member

${ }^{\dagger}$ Visiting Research Scientist II, Atmospheric, Oceanic and Space Sciences

${ }^{\ddagger}$ Associate Research Scientist, Atmospheric, Oceanic and Space Sciences, AIAA Member

${ }^{\S}$ Professor, Aerospace Engineering, AIAA Fellow

TProfessor, Atmospheric, Oceanic and Space Sciences, AIAA Fellow

$\|_{\text {Professor, Electrical Engineering and Computer }}$ Science

Copyright (C)2001 by the American Institute of Aeronautics and Astronautics, Inc. All rights reserved.
}

sults for these problems are given.

\section{Governing Equations}

The governing equations for an ideal, nonrelativistic, compressible plasma may be written in a number of different forms. In primitive variables, the governing equations, which represent a combination of the Euler equations of gasdynamics and the Maxwell equations of electromagnetics, may be written as:

$$
\begin{aligned}
\frac{\partial \rho}{\partial t}+\mathbf{u} \cdot \nabla \rho+\rho \nabla \cdot \mathbf{u} & =0 \\
\rho \frac{\partial \mathbf{u}}{\partial t}+\rho \mathbf{u} \cdot \nabla \mathbf{u}+\nabla p-\mathbf{j} \times \mathbf{B} & =0 \\
\frac{\partial \mathbf{B}}{\partial t}+\nabla \times \mathbf{E} & =0 \\
\frac{\partial p}{\partial t}+\mathbf{u} \cdot \nabla p+\gamma p \nabla \cdot \mathbf{u} & =0
\end{aligned}
$$

where the current density $\mathbf{j}$ and the electric field vector $\mathbf{E}$ are related to the magnetic field B by Ampère's law and Ohm's law, respectively:

$$
\begin{aligned}
\mathbf{j} & =\frac{1}{\mu_{0}} \nabla \times \mathbf{B} \\
\mathbf{E} & =-\mathbf{u} \times \mathbf{B}
\end{aligned}
$$

For one popular class of schemes, the equations are written in a form in which the gasdynamic terms are put in divergence form, and 
the electromagnetic terms in the momentum and energy equations are treated as source terms. This gives:

$$
\begin{aligned}
\frac{\partial \rho}{\partial t}+\nabla \cdot(\rho \mathbf{u}) & =0 \\
\frac{\partial(\rho \mathbf{u})}{\partial t}+\nabla \cdot(\rho \mathbf{u u}+p \mathbf{I}) & =\mathbf{j} \times \mathbf{B} \\
\frac{\partial \mathbf{B}}{\partial t}+\nabla \times \mathbf{E} & =0 \\
\frac{\partial E_{g d}}{\partial t}+\nabla \cdot\left(\mathbf{u}\left(E_{g d}+p\right)\right) & =\mathbf{j} \cdot \mathbf{E}
\end{aligned}
$$

where $E_{g d}$ is the gasdynamic total energy, given by

$$
E_{g d}=\frac{p}{\gamma-1}+\rho \frac{\mathbf{u} \cdot \mathbf{u}}{2}
$$

The fully conservative form of the equations is

$$
\frac{\partial \mathbf{U}}{\partial t}+(\nabla \cdot \mathbf{F})^{T}=0
$$

where $\mathbf{U}$ is the vector of conserved quantities

$$
\mathbf{U}=\left(\begin{array}{c}
\rho \\
\rho \mathbf{u} \\
B \\
E_{m h d}
\end{array}\right)
$$

and $\mathbf{F}$ is a flux diad,

$$
\mathbf{F}=\left(\begin{array}{c}
\rho \mathbf{u} \\
\rho \mathbf{u u}+\left(p+\frac{\mathbf{B} \cdot \mathbf{B}}{2 \mu_{0}}\right) \mathbf{I}-\frac{1}{\mu_{0}} \mathbf{B B} \\
\mathbf{u B}-\mathbf{B} \mathbf{u} \\
\mathbf{u}\left(E_{m h d}+p+\frac{\mathbf{B} \cdot \mathbf{B}}{2 \mu_{0}}\right)-\frac{1}{\mu_{0}}(\mathbf{u} \cdot \mathbf{B}) \mathbf{B}
\end{array}\right)^{T}
$$

where $E_{m h d}$ is the magnetohydrodynamic energy, given by

$$
E_{m h d}=\frac{p}{\gamma-1}+\rho \frac{\mathbf{u} \cdot \mathbf{u}}{2}+\frac{\mathbf{B} \cdot \mathbf{B}}{2 \mu_{0}}
$$

Godunov [1] showed that the fully conservative form, Equation 5, is not symmetrizable. The symmetrizable form may be written as

$$
\frac{\partial \mathbf{U}}{\partial t}+(\nabla \cdot \mathbf{F})^{T}=\mathbf{Q}
$$

where

$$
\mathbf{Q}=-\nabla \cdot \mathbf{B}\left(\begin{array}{c}
0 \\
\mathbf{B} \\
\mu_{0} \\
\mathbf{u} \\
\frac{\mathbf{u} \cdot \mathbf{B}}{\mu_{0}}
\end{array}\right)
$$

Vinokur [2] separately showed that Equation 6 can be derived starting from the primitive form, if no stipulation is made about $\nabla \cdot \mathbf{B}$ in the derivation. Powell [3] showed that this symmetrizable form can be used to derive a Roe-type approximate Riemann solver for solving the MHD equations in multiple dimensions.

The MHD eigensystem arising from Equation 5 or Equation 6 leads to eight eigenvalue/eigenvector pairs. The eigenvalues and associated eigenvectors correspond to an entropy wave, two Alfvén waves, two magnetofast waves, two magnetoslow waves, and an eighth eigenvalue/eigenvector pair that depends on which form of the equations is being solved. This last pair has a zero eigenvalue in the fully conservative case, and an eigenvalue equal to that associated with the entropy wave, in the symmetrizable case. The expressions for the eigenvectors, and the scaling of the eigenvectors, are more intricate than in gasdynamics [4].

\section{Solution Techniques}

Because the MHD equations are a system of hyperbolic conservation laws, many of the techniques that have been developed for the Euler equations can be applied relatively straightforwardly. In particular, the highresolution finite-volume approach [5] (i.e. approximate Riemann solver + limited interpolation scheme + multi-stage time-stepping scheme) is perfectly valid. The Rusanov/LaxFriedrichs approximate Riemann solver can be applied directly; no knowledge of the eigensystem of the MHD equations is required, other than the fastest wave speed in the system. A Roe-type scheme can be constructed, 
but requires more work, because of the complexity of the eigensystem. In addition, an HLLE-type Riemann solver has been derived by Linde [6]; it is less dissipative than the Rusanov/Lax-Friedrichs scheme, but more robust and less computationally intensive than the Roe scheme. Whichever approximate Riemann solver is chosen to serve as the flux function, standard interpolation schemes and limiters can be used to construct a finite-volume scheme.

One added difficulty in solving the MHD equations is that the MHD energy has three components: internal, magnetic and kinetic. Thus, as in gasdynamics, flows with substantially more kinetic energy than internal energy can lead to positivity problems when computing the pressure. Also, in contrast to gasdynamics, regions in which the magnetic field is large can yield similar problems. Conservative and positive HLL-type schemes for MHD have been described by Janhunen [7]. Another alternative, due to Balsara and Spicer [8], is to use a hybrid scheme: both the conservative energy equation and the entropy equations are solved. Close to shock waves the energy equation is used to obtain the correct weak solution, at other places the more robust and positive entropy equation can be used. A variant of this technique has been implemented in our code.

\section{Controlling $\nabla \cdot \mathrm{B}$}

Another way in which the numerical solution of the MHD equations differs from that of the gasdynamic equations is the constraint that $\nabla \cdot \mathbf{B}=0$. Enforcing this constraint numerically, particularly in shock-capturing codes, can be done in a number of ways, but each way has its particular strengths and weaknesses. Only a brief overview is given below; each of the schemes discussed below is explained more fully in the references cited, and Tóth has published a numerical comparison of many of the approaches for a suite of test cases [9]

Brackbill and Barnes [10] first proposed using a Hodge-type projection to the magnetic field. This approach leads to a Poisson equation that must be solved each time the projection takes place:

$$
\begin{aligned}
\nabla^{2} \phi & =\nabla \cdot \mathbf{B} \\
\mathbf{B}_{\text {projected }} & =\mathbf{B}-\nabla \phi
\end{aligned}
$$

The resulting projected magnetic field is divergence-free on a particular numerical stencil, to the level of error of the solution of the Poisson equation. While it is not immediately obvious that the use of the projection scheme in conjunction with the fully conservative form of the MHD equations gives the correct weak solutions, Tóth has proven this to be the case [9]. The projection scheme has several advantages, including the ability to used standard software libraries for the Poisson solution, its relatively straightforward extension to general unstructured grids, and its robustness. It does, however, require solution of an elliptic equation at each projection step; this can be expensive, particularly on distributedmemory machines.

Powell [3, 11] first proposed an approach based on the symmetrizable form of the MHD equations, Equation 6. In this approach, the source term on the right-hand side of Equation 6 is computed at each time step, and included in the update scheme. Discretizing this form of the equations leads to enhanced stability and accuracy, however, there is no stencil on which the divergence is identically zero. In most regions of the flow, the divergence source term is small. However, near discontinuities, it is not guaranteed to be small. In essence, the inclusion of the source term changes what would be a zero eigenvalue of the system to one whose value is $u_{n}$, the component of velocity normal to the interface through which the flux is computed. The scheme is typically referred to as the eight-wave scheme; the eighth wave is corresponds to propagation of jumps in the normal component of the magnetic field. 
The eight-wave scheme can be thought of as a hyperbolic or advective approach to controlling $\nabla \cdot \mathbf{B}$; symmetrizable form of the equations, Equation 6, are consistent with the passive advection of $\nabla \cdot \mathbf{B} / \rho$. The eight-wave scheme is computationally inexpensive, easy to add to an existing code, and quite robust. However, if there are regions in the flow in which the $\nabla \cdot \mathbf{B}$ source term (Equation 7 ) is large, the conservation errors can create problems.

Recently, several approaches have been developed that have combined a Riemannsolver-based scheme with constrainedtransport approach. The constrainedtransport approach, first proposed by Evans and Hawley [12] treated the MHD equations in the gasdynamics/electromagnetic-split form of Equation 4. The grid used was a staggered one, and the $\nabla \cdot \mathbf{B}=0$ constraint was met identically, on a particular numerical stencil. Dai and Woodward [13] and Balsara and Spicer [14] modified the constrained-transport approach by coupling a Riemann-solver-based scheme for the conservative form of the MHD equations, Equation 5 with a constrained-transport approach for the representation of the magnetic field. In their formulations, this required two representations of the magnetic field: a cellcentered one for the Godunov scheme, and a face-centered one to enforce the $\nabla \cdot \mathrm{B}=0$ condition. Tóth [9] subsequently showed that these formulations could be recast in terms of a single cell-centered representation for the magnetic field, through a modification to the flux function used. Advantages of the conservative constrained-transport schemes include the fact that they are strictly conservative and that they meet the $\nabla \cdot \mathbf{B}=0$ constraint to machine accuracy, on a particular stencil. Their primary disadvantage is the difficulty in extending them to general grids. Tóth and Roe [15] made some progress on this front; they developed divergence-preserving prolongation and restriction operators, allowing the use of conservative constrained-transport schemes on h-refined meshes. However, they also showed that the conservative constrained-transport techniques lose their $\nabla \cdot$ B-preserving properties if different cells are advanced at different physical time rates. This rules out the use of local time-stepping. Thus, while for unsteady calculations the cost of the conservative constrained-transport approach is comparable to the eight-wave scheme, for steady-state calculations (where one would typically use local time-stepping), the cost can be prohibitive.

Some of the most recent work on the $\nabla \cdot \mathbf{B}=$ 0 constraint has been related to modifying the eight-wave approach by adding a source term proportional to $\nabla(\nabla \cdot \mathbf{B})$ so that the the divergence satisfies an advection-diffusion equation, rather than a pure advection equation. This technique, due to Linde and Malagoli [16] referred to as diffusive control of $\nabla \cdot \mathbf{B}$, has the same advantages and disadvantages as the eight-wave approach. It is not strictly conservative, but appears to keep the level of $\nabla \cdot \mathrm{B}$ lower than the eight-wave approach does. In other recent work by Dedner et al [17], a generalized Lagrange-multiplier method has been proposed, incorporating the projection approach, the eight-wave approach, and the diffusive-control approach into a single framework.

\section{Validation Studies}

In this section, validation studies are presented that compare the solution methods and $\nabla \cdot \mathbf{B}$ control techiques cited above.

The first test cases are plasma-shock-tube problems. In Figure 1, the results of a one-dimensional plasma-shock-tube problem known as the Brio-Wu problem [18] are presented for three schemes: the eight-wave scheme, based on the symmetrizable form of the equations, the conservative constrained transport scheme, based on the fully conser- 
vative form of the equations, and the nonconservative constrained transport scheme, based on the gasdynamic/electromagnetic split form of the equations. For this problem, the results of the eight-wave scheme and the conservative constrained transport scheme are indistinguishable, and are shown in black. The non-conservative constrained transport scheme results, shown in red, display errors as large as $20 \%$, particularly in the velocity. This is not surprising, of course; the various jumps in the Brio-Wu problem correspond to the Rankine-Hugoniot conditions for a plasma, which differ from those of a gasdynamic shock. Because the equations are not discretized in a divergence form, substantial errors are expected in the presence of non-zero magnetic fields.

One-dimensional plasma-shock-tube problems such as the Brio-Wu problem are popular validation cases for base schemes, but do not test the $\nabla \cdot \mathbf{B}=0$ constraint techniques. This is because, in one dimension, the constraint that $\nabla \cdot \mathbf{B}=0$ degenerates to the constraint that $B_{x}=$ constant. However, rotating a onedimensional problem so that the discontinuities run oblique to the grid yields a problem that can test the $\nabla \cdot \mathbf{B}$ constraint technique. In Figure 2, results are presented for the component of the magnetic field parallel to the direction of motion of the waves (i.e. the analog to $B_{x}$ in the one-dimensional case). The exact solution for this quantity is a constant $\left(B_{\|}=\right.$ $\sqrt{2}$ ); the numerical results differ depending on the $\nabla \cdot \mathbf{B}$ constraint technique. The largest error, on the order of $10 \%$, comes from using the eight-wave scheme in conjunction with the Roe approximate Riemann solver. This is due to the $\nabla \cdot \mathbf{B}$ source term, which is not small in the region of the fast magnetosonic shock in this case, and leads to conservation errors. Surprisingly, the eight-wave scheme in conjunction with the Rusanov/Lax-Friedrichs approximate Riemann solver yields errors that are an order of magnitude smaller. The diffusive $\nabla \cdot \mathbf{B}$ control technique, used in con- junction with the Roe approximate Riemann solver, yields errors on the order of $2 \%$; the conservative constrained-transport technique, yields results that are centered on the correct value, but somewhat oscillatory. It should be noted that only $B_{\|}$is shown here, in part because the errors in other variables are much smaller: the differences in $B_{\perp}$, pressure, density, and $u_{\|}$among the schemes are two orders of magnitude smaller than those in $B_{\|}$; the differences in $u_{\perp}$ among the schemes are more than one order of magnitude smaller than those in $B_{\|}$.

The third validation case is one that is more representative of space-physics calculations. It represents a quasi-steady interaction of the solar wind with Earth's magnetic field. The boundary condition upstream of Earth is a steady plasma flow, with:

- a density of 5 molecules per cubic centimeter,

- a temperature of $180,000 \mathrm{~K}$,

- a velocity of 400 kilometers per second, pointed directly outward from the Sun,

- a magnetic field of 5 nanoTesla, pointed northward.

Earth (including its atmosphere through the ionosphere) is represented as a conducting sphere with an embedded, non-tilted, nonrotating magnetic dipole. These conditions are a simplification of the real situation, in which the flow from the Sun would be unsteady, and the Earth's intrinsic magnetic field more complicated. The calculation is carried out on a three-dimensional, solution-adaptive grid, on a parallel machine. Details of the approach are given by Groth et al [19]. The code can be run first-order or as a second-order MUSCL scheme, using any combination of the solvers and $\nabla \cdot \mathbf{B}$ control techniques mentioned above.

Figure 3 shows the effect of order of accuracy, grid resolution, and solver choice on 

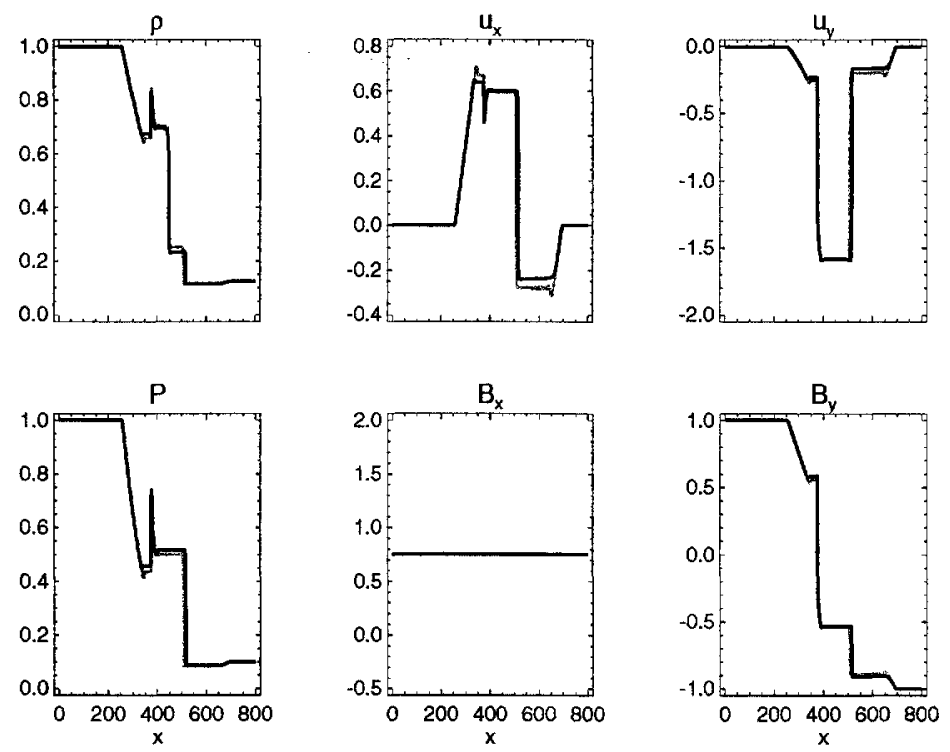

Figure 1: Comparison of Eight-wave and Conservative Constrained Transport Schemes (black) with Non-Conservative Constrained Transport Scheme (red) for Brio-Wu Plasma-Shock-Tube Problem
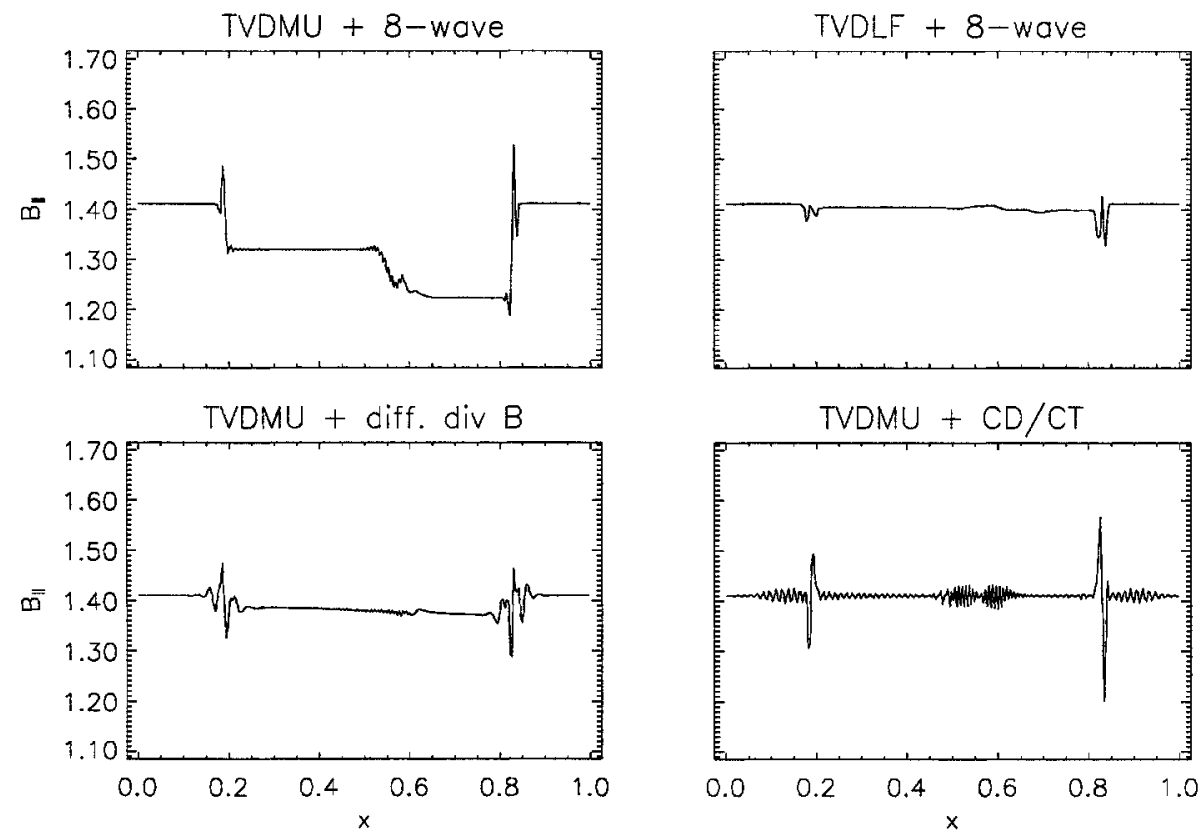

Figure 2: Comparison of Various $\nabla \cdot \mathbf{B}$ Control Techniques on Rotated Plasma-Shock-Tube Problem 
the flow. In the figure, color contours of pressure are superimposed on magnetic field-line traces, in a two-dimensional cut through the noon-midnight plane. A comparison of the upper left and upper right panels of the plot shows the effect of increased grid resolution when the scheme is first-order; the panel on the right resulted from a run with eight times as many cells as that on the left. A comparison of the upper left and lower right panels of the plot shows the effect of increasing the order of accuracy of the scheme; the lower right panel resulted from a run using a secondorder MUSCL scheme. A comparison of the two lower panels shows the effect of different solvers; the Rusanov scheme (on the left) is more dissipative than the Linde scheme (on the right). Of the four panels, the bottom right one is the closest to the grid-converged solution.

Figure 4 shows the effect of $\nabla \cdot \mathbf{B}$ control technique. Four methods - the eight-wave, diffusive control, projection and conservative constrained transport techniques - are compared, using a second-order MUSCL scheme with a Rusanov solver, and a grid with a smallest cell size of a quarter $R_{E}$. Although the grid for this case is relatively coarse, the various $\nabla \cdot \mathbf{B}$ control techniques lead to results that differ by only $1-2 \%$. The relative cost depends on implementation, but the eight-wave and diffusive-control techniques are the least expensive, the projection scheme somewhat more (because of the elliptic step each time the magnetic field is projected) and the constrained transport is substantially more expensive (approximately a factor seven over the eight-wave scheme) because of the inability to use local time-stepping in this steady problem.

\section{Semi-Relativistic Plasmas}

While the solar-wind speed remains nonrelativistic in the solar system, the intrinsic magnetic fields of several planets in the solar system are high enough, and the density of the solar wind low enough, that the Alfvén speed,

$$
V_{A}=\sqrt{\frac{\mathbf{B} \cdot \mathbf{B}}{\mu_{0} \rho}}
$$

can reach appreciable fractions of the speed of light. In the case of Jupiter, the Alfvén speed in the vicinity of the poles is of order ten! Even Earth has a strong enough intrinsic magnetic field that the Alfvén speed reaches twice the speed of light in Earth's near-auroral regions.

For these vicinities, solving the nonrelativistic ideal MHD equations does not make sense. Having waves in the system propagating faster than the speed of light, besides being non-physical, causes a number of numerical difficulties. However, solving the fully relativistic MHD equations is overkill. What is called for is a semi-relativistic form of the equations, in which the flow speed and acoustic speed are non-relativistic, but the Alfvén speed can be relativistic. A derivation of these semi-relativistic equations from the fully relativistic equations is given in Gombosi et al [20]; the final result is presented here.

The semi-relativistic ideal MHD equations are of the form

$$
\frac{\partial \mathbf{U}_{s r}}{\partial t}+\left(\nabla \cdot \mathbf{F}_{s r}\right)^{T}=0
$$

where the state vector, $\mathbf{U}_{s r}$, and the flux diad, $\mathbf{F}_{s r}$, are

$$
\begin{gathered}
\mathbf{U}_{s r}=\left(\begin{array}{c}
\rho \\
\rho \mathbf{u}+\frac{1}{c^{2}} \mathbf{S}_{A} \\
\mathbf{B} \\
\frac{\rho \mathbf{u} \cdot \mathbf{u}}{2}+\frac{p}{\gamma-1}+e_{A}
\end{array}\right) \\
\mathbf{F}_{s r}=\left(\begin{array}{c}
\rho \mathbf{u} \\
\rho \mathbf{u} \mathbf{u}+p \mathbf{I}+\mathbf{P}_{A} \\
\mathbf{u} \mathbf{B}-\mathbf{B} \mathbf{u} \\
\left(\frac{\rho \mathbf{u} \cdot \mathbf{u}}{2}+\frac{\gamma p}{\gamma-1}\right) \mathbf{u}+\mathbf{S}_{A}
\end{array}\right)^{T}
\end{gathered}
$$

In the above,

$$
\mathbf{S}_{A}=\frac{1}{\mu_{0}}(\mathbf{E} \times \mathbf{B})
$$



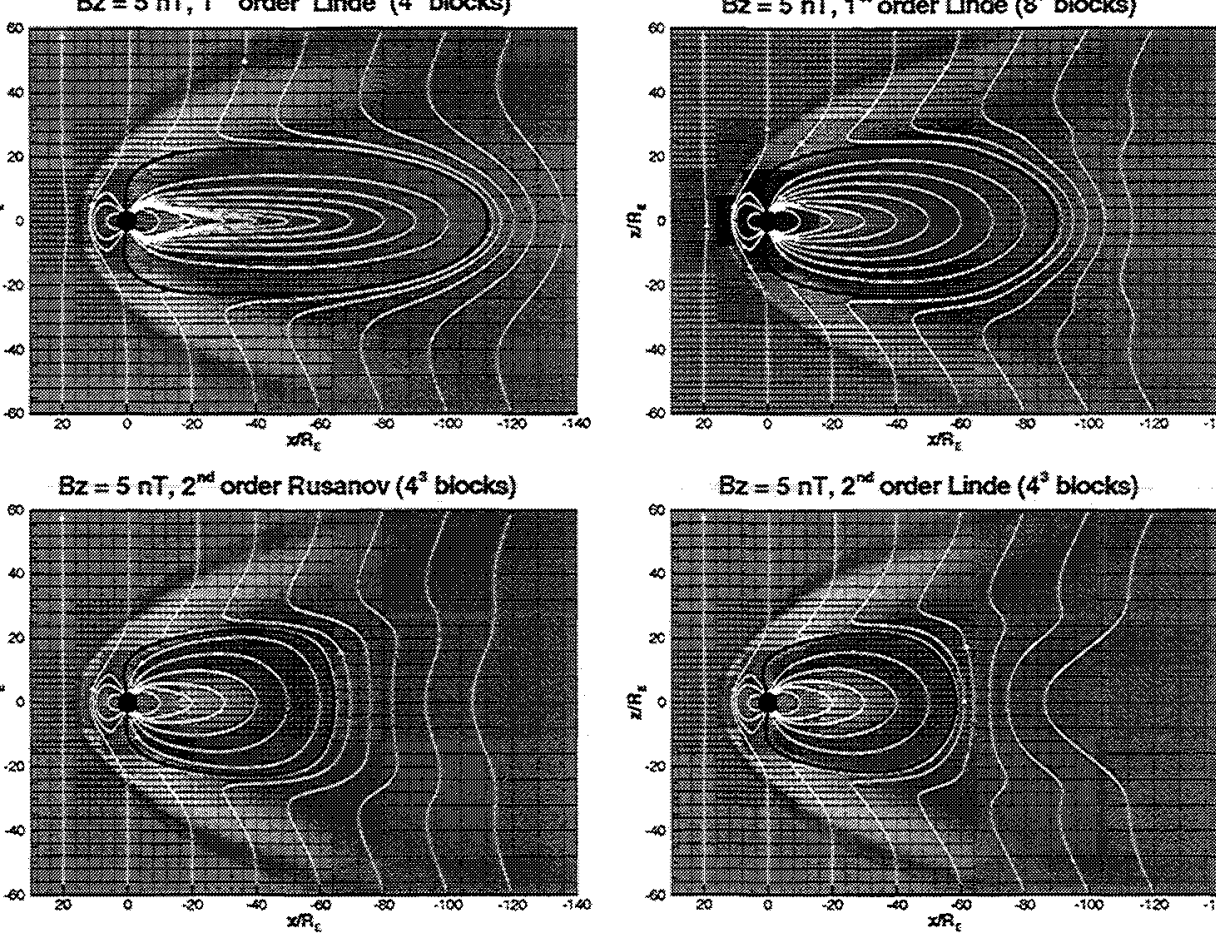

Figure 3: Comparison of Various Solvers and Resolutions for Earth's Magnetosphere Simulation
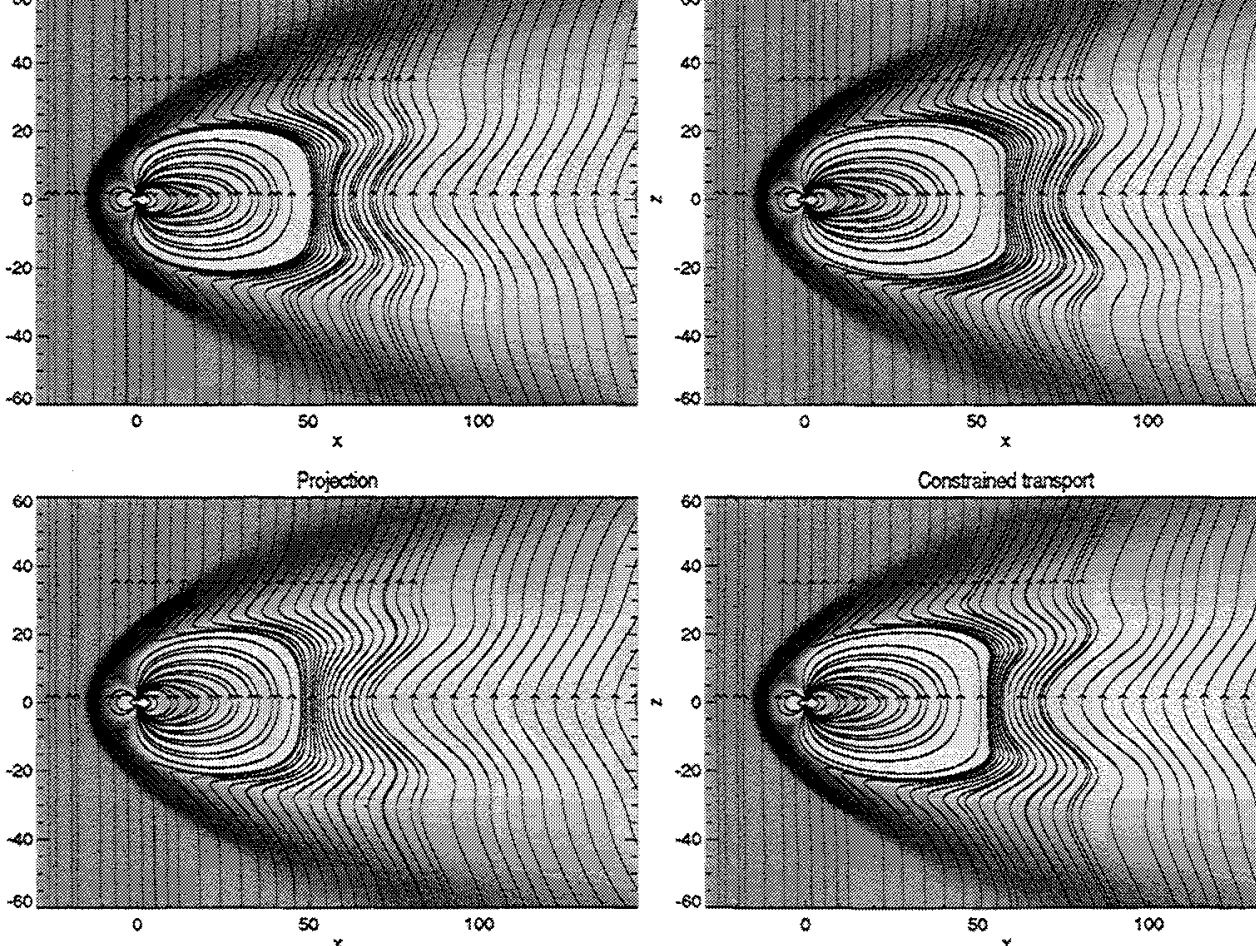

Figure 4: Comparison of $\nabla \cdot \mathbf{B}$ Control Techniques for Earth's Magnetosphere Simulation 


$$
\begin{aligned}
e_{A} & =\frac{1}{2 \mu_{0}}\left(B^{2}+\frac{1}{c^{2}} E^{2}\right) \\
\mathbf{P}_{A} & =e_{A} \mathbf{I}-\frac{1}{\mu_{0}} \mathbf{B} \mathbf{B}-\frac{1}{\mu_{0} c^{2}} \mathbf{E} \mathbf{E}
\end{aligned}
$$

are the Poynting vector, the electromagnetic energy density, and the electromagnetic pressure tensor, respectively. The electric field $\mathbf{E}$ is related to the magnetic field $\mathbf{B}$ by Ohm's law, Equation 3.

This new system of equations has wave speeds that are limited by the speed of light; for strong magnetic fields, the modified Alfvén speed (and the modified magnetofast speed) asymptote to $c$. The modified magnetoslow speed asymptotes to $a$, the acoustic speed. This property offers the possibility of a rather tricky convergence-acceleration technique, first suggested by Boris [21]; the wave speeds can be lowered, and the stable timestep thereby raised, by artificially lowering the value taken for the speed of light.

The equations above are valide in physical situations in which $V_{A}>c$. A slight modification yields a set of equations, the steady-state solutions of which are independent of the value taken for the speed of light. Defining the true value of the speed of light to be $c_{0}$, to distinguish it from the artificially lowered speed of light, $c$, the equations are:

$$
\frac{\partial \mathbf{U}_{s r}}{\partial t}+\left(\nabla \cdot \mathbf{F}_{s r}\right)^{r}=\mathbf{Q}_{c_{0}}
$$

where the state vector, $\mathbf{U}_{s r}$, and the flux diad, $\mathbf{F}_{s r}$, are as defined above, and the new source term is

$$
\mathbf{Q}_{c_{0}}=\frac{1}{\mu_{0}}\left(\frac{1}{c_{0}^{2}}-\frac{1}{c^{2}}\right) \mathbf{E} \nabla \cdot \mathbf{E}
$$

An implementation of the semi-relativistic equations has been made. It is based on the Rusanov/Lax-Friedrichs approximate Riemann solver; the Roe scheme for the semirelativistic equations would be quite a mess, due to the complicated expressions for the eigenvalues and eigenvectors. The eight-wave scheme is used to control $\nabla \cdot \mathbf{B}$.
Figure 5 shows steady-state results for the same magnetosphere case as above. Two runs were made: a non-accelerated run, with $c=c_{0}$ (and painfully small time steps); and an accelerated run, with $c=c_{0} / 200$. The accelerated run took an order of magnitude less time, due to the higher stable time step. The test confirmed that the two solutions are the same to the level of truncation error.

\section{Acknowledgments}

This work was supported by NSF KDI grant NSF ATM-9980078, NSF CISE grant ACI-9876943, and NASA IASRP grant NAG5-9406. G. Tóth is partially supported by a postdoctoral fellowship (D 25519) from the Hungarian Science Foundation (OTKA).

\section{References}

[1] S. K. Godunov, "Symmetric form of the equations of magnetohydrodynamics (in Russian)," in Numerical Methods for Mechanics of Continuum Medium, vol. 1, pp. 26-34, Siberian Branch of USSR Acad. of Sci., 1972.

[2] M. Vinokur, "A rigorous derivation of the MHD equations based only on Faraday's and Ampére's laws." Presentation at LANL MHD Workshop, 1996.

[3] K. G. Powell, "An approximate Riemann solver for magnetohydrodynamics (that works in more than one dimension)," Tech. Rep. 94-24, Inst. for Comput. Appl. in Sci. and Eng., NASA Langley Space Flight Center, Hampton, Va., 1994.

[4] P. L. Roe and D. S. Balsara, "Notes on the eigensystem of magnetohydrodynamics," SIAM J. Appl. Math., vol. 56, pp. 57-67, Feb. 1996.

[5] B. van Leer, "Towards the ultimate conservative difference scheme. V. A second- 

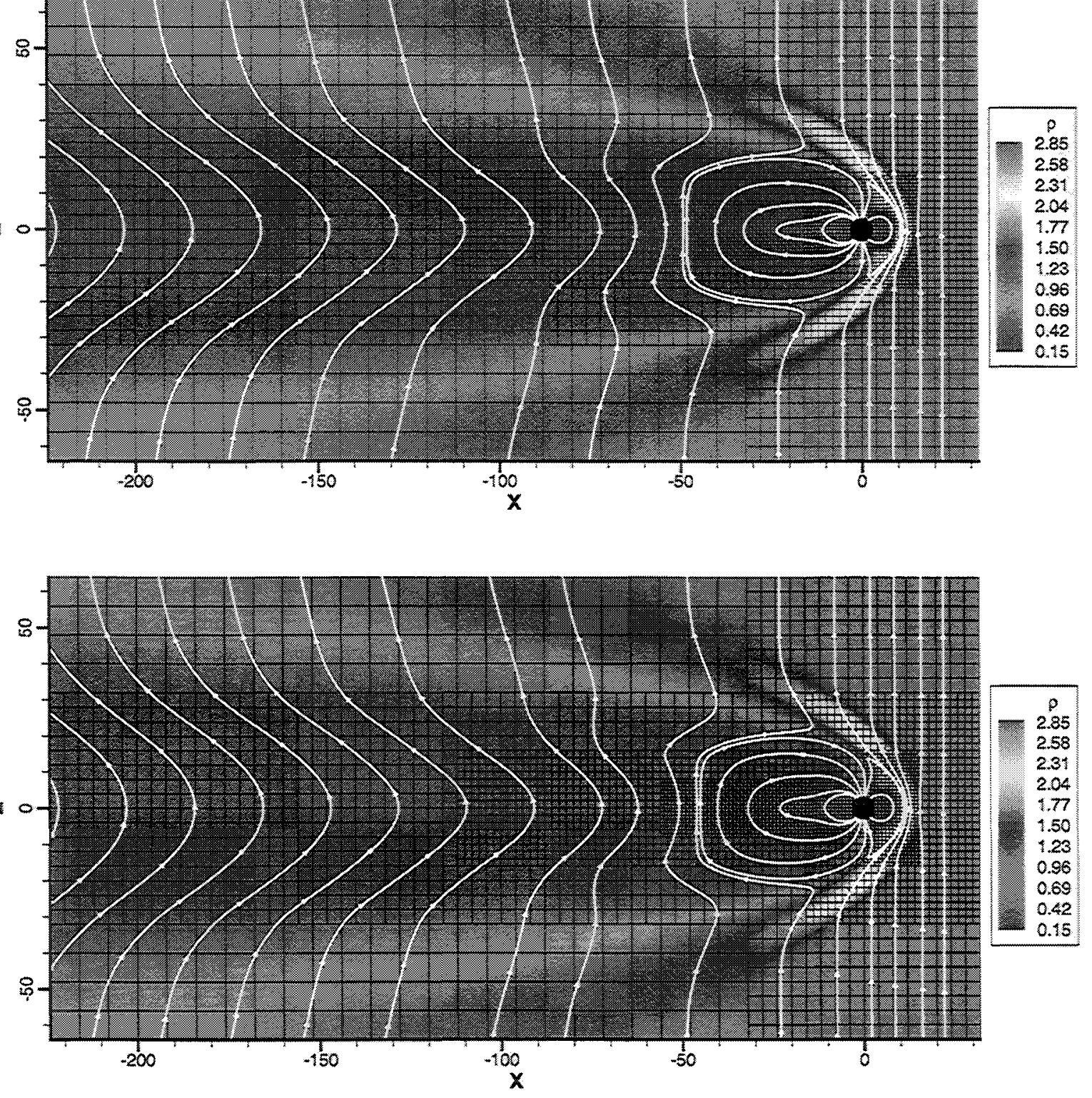

Figure 5: Comparison of Standard $\left(c=c_{0}\right)$ and Accelerated $\left(c=c_{0} / 200\right)$ Run Results for Magnetosphere Case 
order sequel to Godunov's method," $J$. Comput. Phys., vol. 32, pp. 101-136, 1979.

[6] T. J. Linde, A Three-Dimensional Adaptive Multifluid MHD Model of the Heliosphere. PhD thesis, Univ. of Mich., Ann Arbor, May 1998.

[7] P. Janhunen, "A positive conservative method for magnetohydrodynamics based on HLL and roe methods," J. Comput. Phys., vol. 160, pp. 649-661, 2000.

[8] D. S. Balsara and D. S. Spicer, "Maintaining pressure positivity in magnetohydrodynamic simulations," J. Comput. Phys., vol. 148, pp. 133-148, 1999.

[9] G. Tóth, "The $\nabla \cdot \mathbf{B}$ constraint in shock capturing magnetohydrodynamic codes," J. Comput. Phys., vol. 161, pp. 605-652, 2000 .

[10] J. Brackbill and D. Barnes, "The effect of nonzero $\nabla \cdot \mathbf{B}$ on the numerical solution of the magnetohydrodynamic equations," J. Comput. Phys., vol. 35, pp. 426-430, 1980 .

[11] K. G. Powell, P. L. Roe, T. J. Linde, T. I. Gombosi, and D. L. D. Zeeuw, "A solution-adaptive upwind scheme for ideal magnetohydrodynamics," J. Comput. Phys., vol. 154, pp. 284-309, Sept. 1999.

[12] C. R. Evans and J. F. Hawley, "Simulation of magnetohydrodynamic flows: A constrained transport method," Astrophysical Journal, vol. 332, pp. 659-677, 1988.

[13] W. Dai and P. R. Woodward, "A simple finite difference scheme for multidimensional magnetohydrodynamic equations," J. Comput. Phys., vol. 142, p. 331, 1998.
[14] D. S. Balsara and D. S. Spicer, "A staggered mesh algorithm using high order Godunov fluxes to ensure solenoidal magnetic fields in magnetohydrodynamic simulations," J. Comput. Phys., vol. 149, pp. 270-292, 1999.

[15] G. Tóth and P. L. Roe, "Divergence- and curl-preserving prolongation and restriction formulae," J. Comput. Phys., 2000. submitted.

[16] T. Linde and A. Malagoli, "On local $\nabla$. B control in ideal MHD simulations," $J$. Comput. Phys., 2000. submitted.

[17] A. Dedner, F. Kemm, D. Kröner, C.D. Munz, T. Schnitzer, and M. Wesenberg, "Hyperbolic divergence cleaning for the MHD equations," J. Comput. Phys., 2001. submitted.

[18] M. Brio and C. C. Wu, "An upwind differencing scheme for the equations of ideal magnetohydrodynamics," J. Comput. Phys., vol. 75, pp. 400-422, 1988.

[19] C. P. T. Groth, D. L. De Zeeuw, K. G. Powell, T. I. Gombosi, and Q. F. Stout, "A parallel solution-adaptive scheme for ideal magnetohydrodynamics," in Proc. 14th AIAA Computational Fluid Dynamics Conference, (Norfolk, Virginia), AIAA Paper No. 99-3273, June 1999.

[20] T. I. Gombosi, G. Tóth, D. L. D. Zeeuw, K. C. Hansen, K. Kabin, and K. G. Powell, "Semi-relativistic magnetohydrodynamics and physics-based convergence acceleration," J. Comput. Phys., 2001. submitted.

[21] J. P. Boris, "A physically motivated solution of the Alfvén problem," Tech. Rep. NRL Memorandum Report 2167, Naval Research Laboratory, Washington, D.C., 1970 . 